\title{
Quality control of Ebro magnetic observatory using momentary values
}

\author{
J. J. Curto and S. Marsal \\ Observatori de l'Ebre, CSIC-Universidad Ramón Llull, Spain
}

(Received March 27, 2007; Revised October 24, 2007; Accepted October 24, 2007; Online published November 30, 2007)

\begin{abstract}
Intercomparison of momentary values from observatories across Europe can be used as a test of reliability for a particular magnetic station in this area, as well as the whole network. The method presented by Voppel at the IAGA Assembly of Grenoble (1975) and developed by Schulz and Voppel at the IAGA Assembly of Edinburgh (1981) is based on simultaneous measurements taken at 02:00 UT, which coincides with the period least disturbed by $\mathrm{Sq}$ associated currents on central European longitudes. A selected list of ten least disturbed days per month is provided by the Niemegk (initially Wingst) Geomagnetic Observatory which gathers the corresponding momentary values from the collaborating institutions. This method can be applied to detect fluctuations or jumps in geomagnetic standards. Independent techniques, like linear regression and axial intercept of the standard deviation of the mutual differences of monthly mean values, have been applied to the magnetic elements of Ebro Observatory (EBR) for the period 1997-2001. These tools give results in good agreement amongst them, and most of the coefficients are similar to those obtained for the most significant observatories of the network. No jumps or trends in data are observed, indicating excellent performance of EBR.
\end{abstract}

Key words: Momentary values, quality control, magnetic observatories.

\section{Introduction}

One of the tasks of a magnetic observatory is to keep track of the geomagnetic secular variation (SV). Therefore magnetic observatories are intended to operate over long periods of time (decades). Changes to the surrounding environment of the absolute measurement buildings or the pier stability of the sensors in the variation room (tilting) can compromise the baseline measurements required for tracking SV (Jankowski and Sucksdorff, 1996). An effort to assess the intrinsic limitations of the instruments has been accomplished (Stuart, 1972; Forbes, 1987; Marsal and Torta, 2007). A major difficulty of any observatory is the proper operation of the magnetic instruments. To ensure continuity of operation between observers a manual of operating instructions written by Gaya-Piqué and Torta (2000) is used at EBR. Even, when on occasion, the measurements are not done under appropriate conditions; some corrections are performed (Curto and Sanclement, 2001). Finally, attending international workshops with standards intercomparison is a good practice to detect instrument malfunctions and observer bias. In spite of this, sometimes unknown causes produce base values which differ from their real value, introducing errors that are very difficult to detect with data from only one observatory.

In 1955, five Central European magnetic observatories were proposed to compare their data on the basis of momentary values (Schulz and Beblo, 1996). The aim of this inter-comparison was to provide a quality test for each collaborating observatory, as well as for the whole network.

Copyright (c) The Society of Geomagnetism and Earth, Planetary and Space Sciences (SGEPSS); The Seismological Society of Japan; The Volcanological Society of Japan; The Geodetic Society of Japan; The Japanese Society for Planetary Sciences; TERRAPUB.
As time went on, more observatories joined the program, covering nowadays most of the European zone.

The momentary values arise from the different elements of the magnetic field, namely $D, H, Z$ (and $F$ in some cases). They are simultaneously taken at 02:00 UT, since this time is assumed to coincide with the least disturbed period by $\mathrm{Sq}$ on central European longitudes. The benefit of taking momentary values at this hour is that they are essentially free from the bias produced by the daily magnetic variation $(\mathrm{Sq})$, which has a strong dependence on latitude and local time. This bias would mask small jumps when comparing observatories at different latitudes and longitudes. A list of ten least disturbed days per month is provided by the Niemegk (initially Wingst) Geomagnetic Observatory. With this selection, magnetic disturbances of external origin are minimized. The result obtained is a data set consisting of the corresponding momentary values from all collaborating institutions.

Inter-comparison between these values can be applied to detect fluctuations or jumps in the geomagnetic base-lines, as well as incorrect adjustments, magnetic impurities in the vicinity of the pier, or electromagnetic interferences.

To check Ebro Observatory accuracy and stability we performed an inter-comparison with data compiled during the 1997-2001 period. The list of the observatories used here can be found in Table 1 .

In Fig. 1, a map of Europe displays the positions of the magnetic observatories used in this study. Ebro observatory (EBR), located in the SW corner of this area has few neighbours. This may lead to problems of representation for phenomena with gradients not aligned to the main axis of the distribution. 
Table 1. Magnetic observatories used in this study with their position relative to EBR.

\begin{tabular}{rcrrr}
\hline ordinal & Observatory & $\Delta$ Distance & \multicolumn{1}{c}{$\Delta$ LAT } & \multicolumn{1}{c}{$\Delta$ LON } \\
& code & $(\mathrm{km})$ & $\left({ }^{\circ}\right)$ & \multicolumn{1}{c}{$\left.{ }^{\circ}\right)$} \\
\hline 1 & WNG & 1574 & 12.923 & 8.580 \\
2 & BFE & 1839 & 14.805 & 11.179 \\
3 & WIT & 1413 & 11.997 & 6.174 \\
4 & NGK & 1558 & 11.252 & 12.182 \\
5 & DOU & 1079 & 9.277 & 4.102 \\
6 & FUR & 1182 & 7.345 & 10.784 \\
7 & WIK & 1501 & 7.445 & 15.825 \\
8 & THY & 1549 & 6.080 & 17.400 \\
9 & SUA & 2143 & 3.860 & 25.760 \\
10 & GCK & 1709 & 3.813 & 20.274 \\
11 & PAG & 1975 & 1.695 & 23.684 \\
12 & ISK & 2395 & 0.243 & 28.569 \\
13 & BDV & 1404 & 8.260 & 13.522 \\
14 & BEL & 1974 & 11.017 & 20.299 \\
15 & HRB & 1609 & 7.053 & 17.697 \\
16 & NUR & 2744 & 19.688 & 24.162 \\
17 & AQU & 1082 & 1.563 & 12.824 \\
18 & ESK & 1635 & 14.497 & -3.693 \\
19 & HAD & 1194 & 10.175 & -4.976 \\
20 & LER & 2151 & 19.313 & -1.676 \\
21 & CLF & 813 & 7.203 & 1.767 \\
22 & HLP & 2047 & 13.788 & 18.322 \\
23 & LOV & 2389 & 18.525 & 17.334 \\
24 & EBR & 0.000 & 0.000 \\
25 & DOB & 2434 & 21.253 & 8.624 \\
26 & VAL & 1483 & 11.113 & -10.743 \\
27 & SPT & 436 & -1.273 & -4.843 \\
28 & MAB & 1128 & 9.478 & 5.189 \\
\hline & & & & \\
\hline
\end{tabular}

\section{Procedure}

\subsection{Analysis of long term trends}

For each magnetic element of each observatory we have made use of the monthly means of the momentary values at 02:00 UT reported by Niemegk. With this data, the differences have been calculated by subtracting each participating observatory mean from the respective mean Ebro value:

$$
\Delta e=\langle e\rangle_{\mathrm{EBR}}-\langle e\rangle_{\mathrm{XXX}}
$$

where $\langle e\rangle_{\mathrm{EBR}}$ and $\langle e\rangle_{\mathrm{XXX}}$ are the monthly means of the momentary values for the " $e$ " magnetic element, and XXX stands for the 3 letter IAGA code of a magnetic observatory. The temporal evolution of $\Delta e$ over five years is shown in Fig. 2 where, in order to facilitate the comparison, traces have been normalised to an artificial offset so they start in a common place.

We observe the following traits:

- $\Delta e$ for each observatory has a different gradient due to its individual secular variation.

- For a given magnetic effect, similar peaks appear simultaneously in several observatories, in particular those of the $Z$ component. Looking in detail: some of them are magnified at subauroral observatories (Nurmijärvi (NUR), Lovo (LOV), Dombas (DOB) and Lerwick (LER)); whereas in lower latitude observatories (San Pablo-Toledo (SPT)) or western longitudes

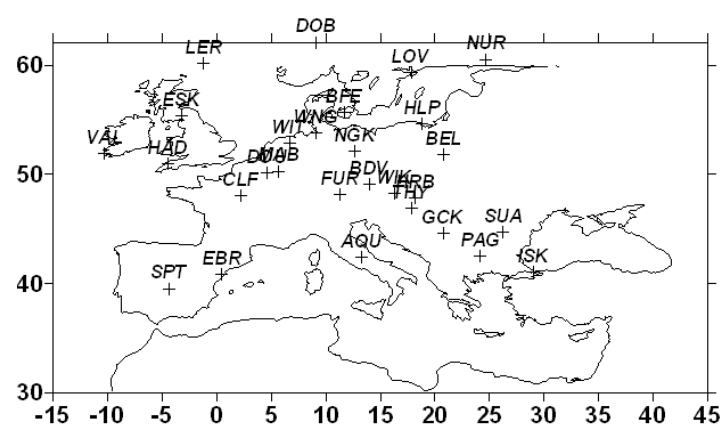

Fig. 1. Magnetic observatories over Europe used in this study.

(Valentia (VAL)) these peaks appear with an opposite sense. These phenomena must be linked to processes such as magnetospheric disturbances, ring current, or others.

- Observatories in the Ebro neighbourhood such as Chambon-la-Foret (CLF), L'Aquila (AQU), or San Pablo-Toledo (SPT) in most cases show relatively small slopes.

- There are anomalous traces which don't correlate with their neighbouring observatories. This is the case of Istanbul-Kandilli (ISK) for 1997 for the $H$ and $Z$ components, Tihany (THY) for 1998, which presents a jump in $D, H$ and $Z$ components, and finally Surlari (SUA) for 2000 in $D$ and for 2001 in $D, H$ and $Z$. The magnetic elements of these observatories for the mentioned years should not be taken into consideration in the statistics. Secular variation has a large spatial scale because its source is far away from the stations as compared with the distance between neighbouring observatories, so large differences between them should be considered as anomalous values and hence disregarded.

Traces of $\Delta e$ follow radial lines and there are no evidences of global jumps or bending, which would point to EBR malfunction.

Secular variation (SV) can be expressed with models (Parkinson, 1983; Bloxham and Gubbins, 1985). One of the most important models of SV is the International Geomagnetic Reference Field (IGRF) (Macmillan and Maus, 2005). It is sponsored by IAGA and is kept updated by a continuous effort of many researchers. Maps of Europe showing isolines of SV based on the IGRF model for Declination, Horizontal intensity, Vertical intensity and Total Force for 2000 are displayed in Fig. 3.

In order to evaluate to what extent the slopes found in Fig. 2 represent secular variation, and knowing the preferred direction of variation of SV according to the IGRF model, we now take as the binning parameter the projection of the distance along the directions marked by the gradient of the SV (big arrows in Fig. 3). Results are displayed in Fig. 4. Correlation coefficients $(r)$ indicate a good linear correlation. The lines of best fit pass very close to the centre of coordinates represented here with an open circle. This confirms the agreement of the long term trends experienced at Ebro with those of the network. 

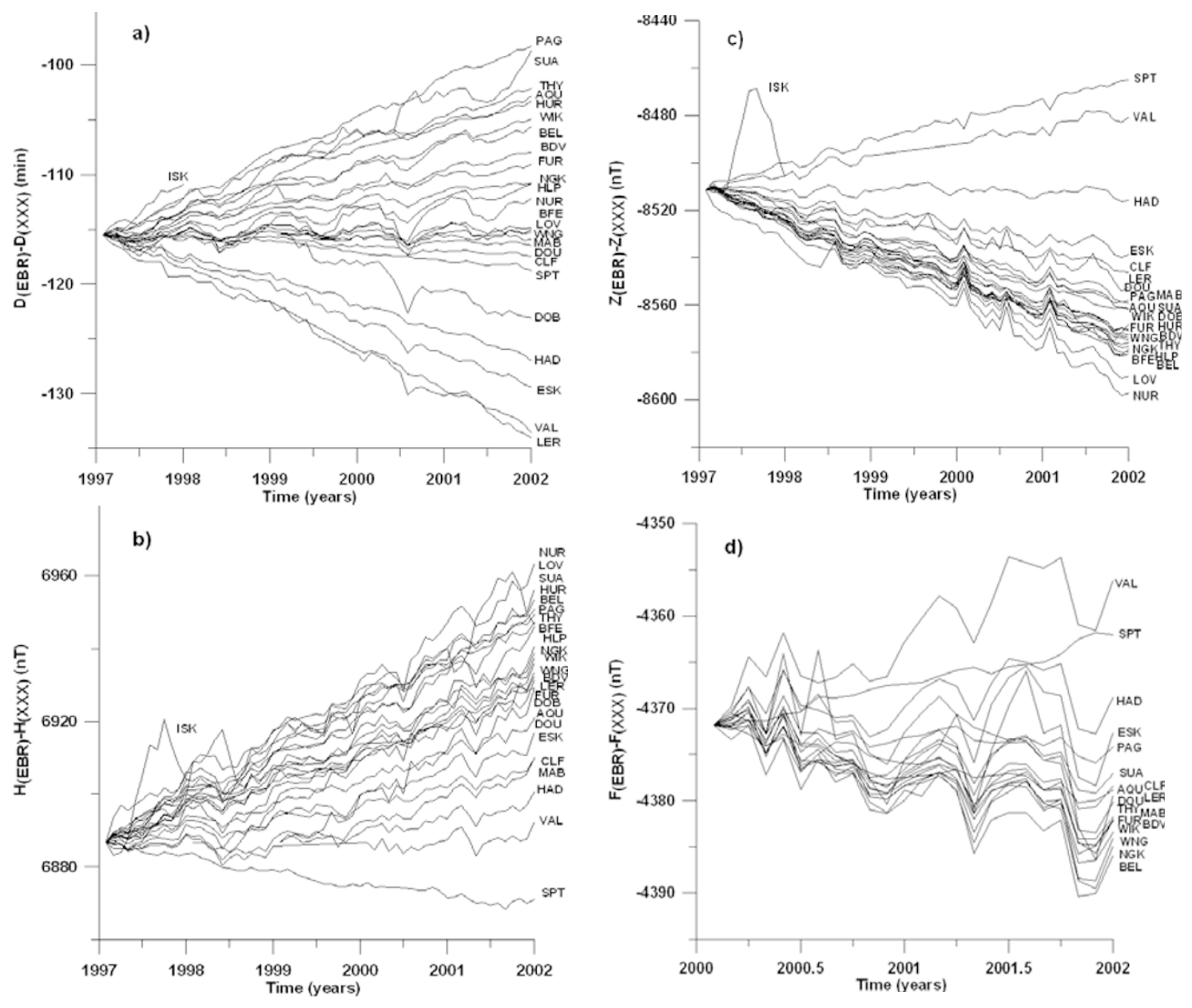

Fig. 2. Temporal variation of the differences between EBR and other observatories ( $\Delta e$ ) for the magnetic components; a) $D$, b) $H$, c) $Z$, and d) $F$.
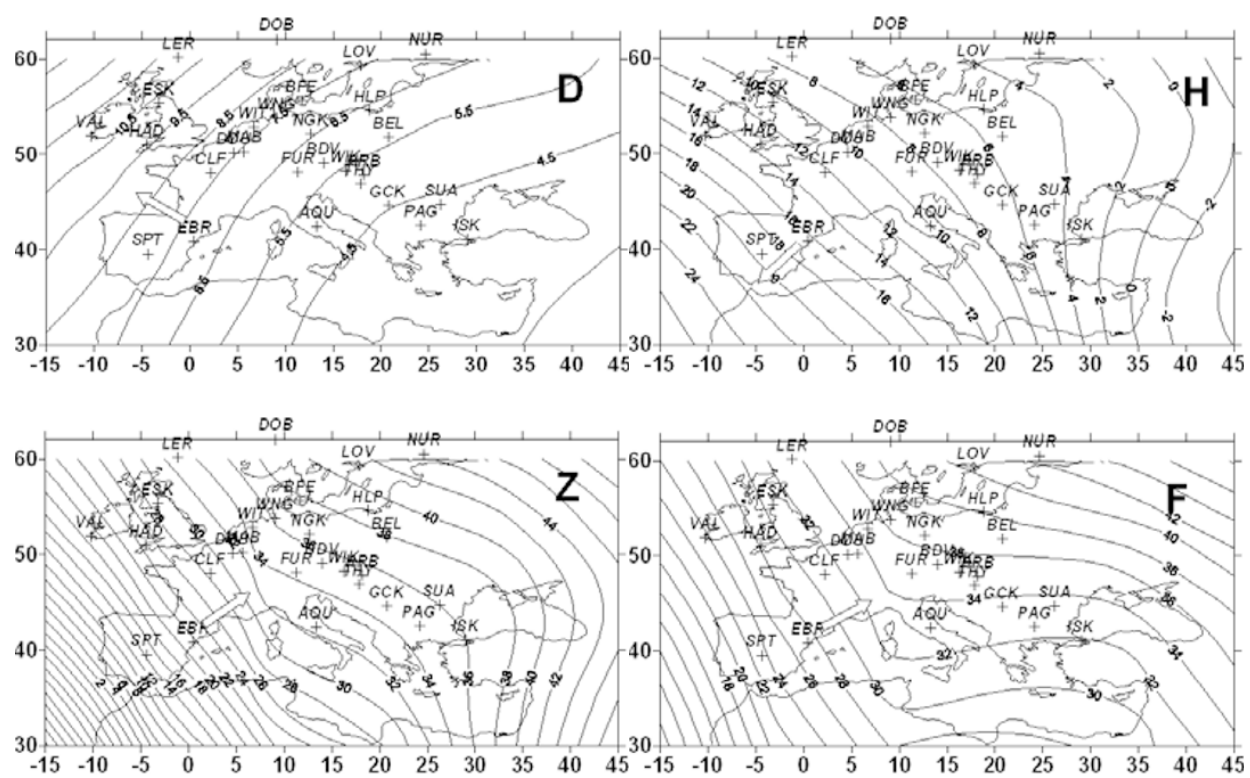

Fig. 3. Secular variation of declination, horizontal intensity, vertical intensity and total force for 2000 , based on the IGRF model. Units for the isolines are nT/year for $H, Z$ and $F$ and min/year for $D$. Big arrows represent the main direction of the gradient in the EBR region.

Some points departing from the fit could announce some small malfunctions in that observatory or that the projection of their coordinates done accordingly to the local gradient at EBR should not be valid there, a probable cause for observatories located far away from EBR. This could be observed in $F$ component, where the magnitude of the correlation coefficient, $r$, is lower.

To gain visual resolution, the derivative with respect to 


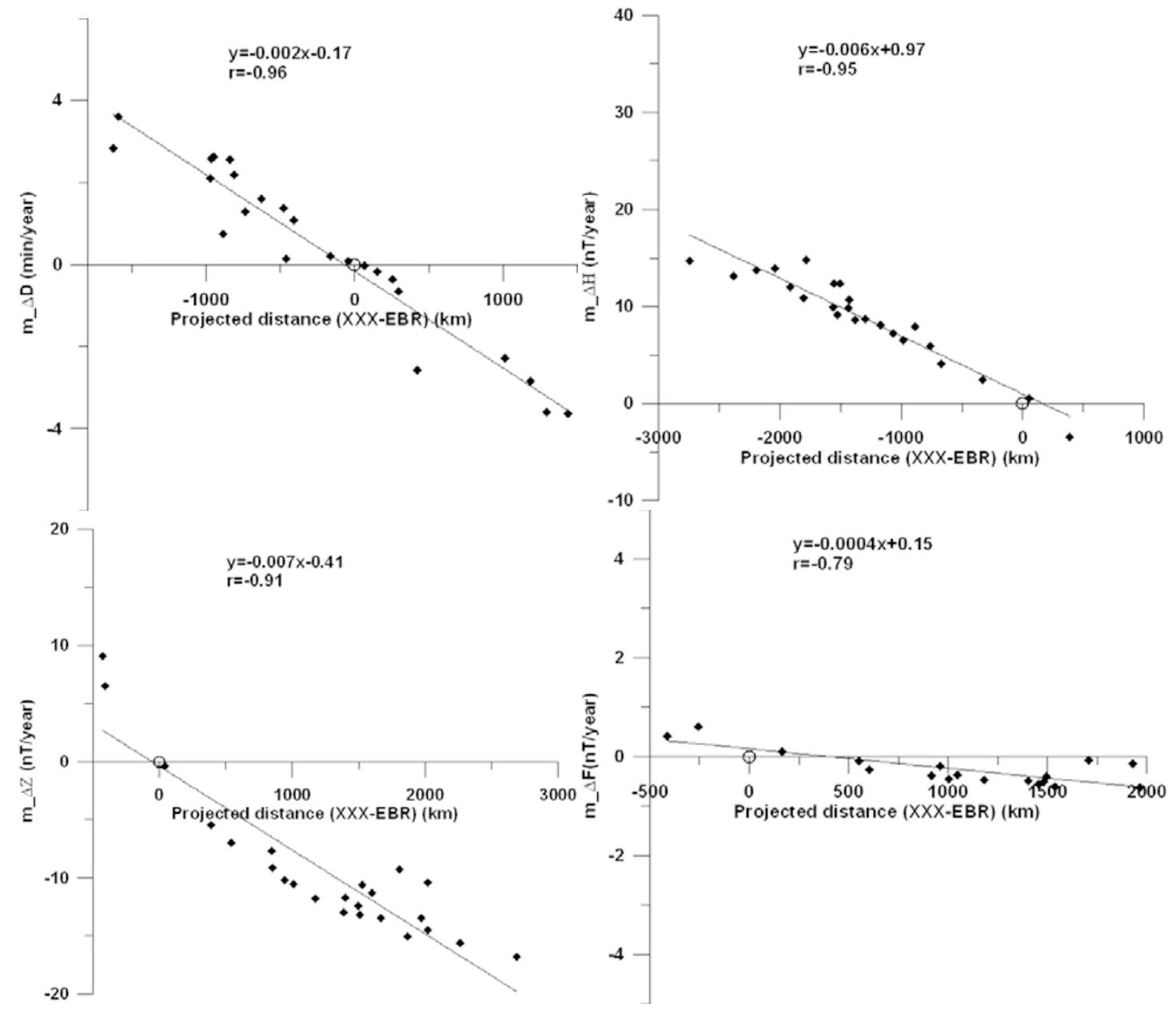

Fig. 4. Slope versus projected distance for $D$ (upper-left), $H$ (upper-right), $Z$ (bottom-left) and $F$ (bottom-right).

$\Delta \mathrm{e}=\langle\mathrm{eebr}\rangle-<\mathrm{e} x \mathrm{x}\rangle$

1)

2)

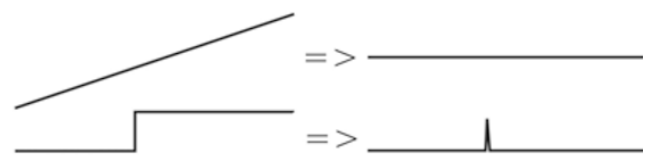

3)

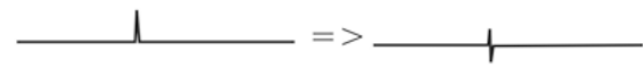

4)

5)

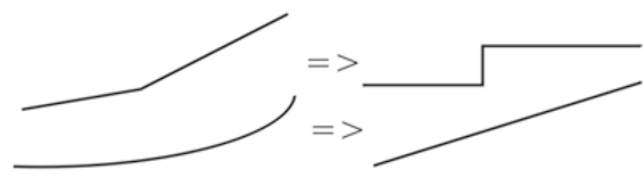

Fig. 5. Some cases of magnetic differences (left) and the appearance of their temporal derivative (right). 1) SV, 2) step due to the introduction of a ferromagnetic object in the neighbourhood of the absolute hut or electronic or mechanical jumps in the standards, 3) isolated anomalous value, 4) reallocation of the absolute magnetometer or progressive un-levelling, 5) magnetization or magnet ageing of the sensor.

time of $\Delta e$ can be plotted. The expected slope due to differences of SV between EBR and another observatory would then be represented as a horizontal line (Fig. 5(1)). A step in EBR would produce here a peak (triangle function) appearing simultaneously in all traces (Fig. 5(2)). An anomalous value would produce a double peak (up-and-down or downand-up) (Fig. 5(3)). A change in tendency (slope) in EBR would be represented here as a step function in most of the lines (Fig. 5(4)) and, finally, an acceleration in EBR would be represented here as a continuous slope (Fig. 5(5)).

Traces of the temporal derivative of the differences between Ebro and the other observatories are plotted in Fig. 6.

None of the long term malfunctions appear here in any element. However, there are some double peaks (up-anddown) (e.g. in $Z$ component) which would correspond to punctual anomalous values. Again, careful inspection shows that those stations to the East of Ebro (CLF, AQU, etc.) systematically have different peak senses from those to the West (SPT, VAL) (Fig. 6(e)). Many of these phenomena may be related to natural noise due to non-quiet magnetic conditions, such as ionospheric local meridional currents closing a global circuit, or as a result of anomalous magnetic transient variations related to the presence of conductivity heterogeneities in the crust and/or lithosphere. 2.2 Analysis of the coherence of short term variations

The physics driving the distribution of the short term variations is not at all the same as that driving the distribution of the secular variation. The sources of short term variations are usually located in the ionosphere (diurnal variation $(\mathrm{Sq})$, lunar variation $(\mathrm{Lq})$, solar flare effects (sfe)), in the magnetosphere (magnetic pulsations, ULF) or in both 

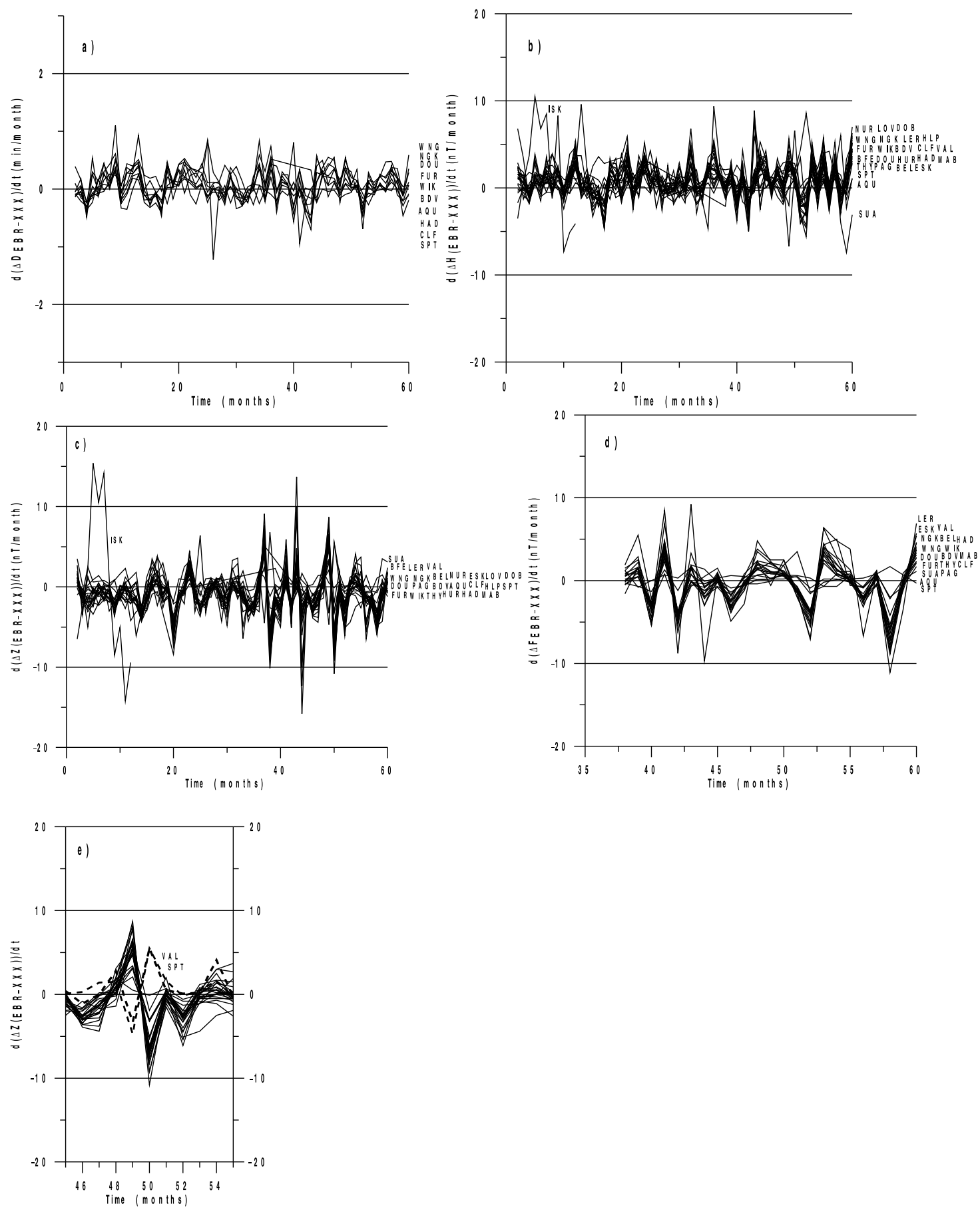

Fig. 6. Derivative of the differences between Ebro and the other observatories for a) $D$, b) $H$, c) $Z$ and d) $F$. e) Detail of Fig. 6(c) showing a double peak. Most of the observatories to the East of EBR have an up-to-down sense, whereas Western observatories (SPT, VAL) present the opposite sense.

(sudden storm commencements (SSC), substorms) (Parkinson, 1983), and their manifestation sometimes follow irregular patterns.

2.2.1 Test of coherence To check the response of Ebro observatory to "short term variations" in relation to those of the network, another analysis can be performed using the standard deviation $(\sigma)$ of the differences of the magnetic elements between Ebro and each one of the remaining observatories (Schulz and Gentz, 1998). This will provide the degree of representation of Ebro observatory within the network.

Taking the monthly means of the differences computed in the previous section, by way of first order regression models estimated from the five neighbouring year values secular variation (SV) is removed, one can evaluate the annual standard deviation of $\Delta e$ for each observatory $\left(\sigma_{-} \Delta e\right)$. Figures 7(a) and 7(b) show $\sigma_{-} \Delta e$ versus absolute value of distance, longitude or latitude relative to EBR. Again a linear fit can be established as:

$$
\sigma_{-} \Delta e(x)=A+B^{*}|x|
$$

where $x$ is either distance, latitude or longitude difference with respect to Ebro, depending on each case. Afterwards, 

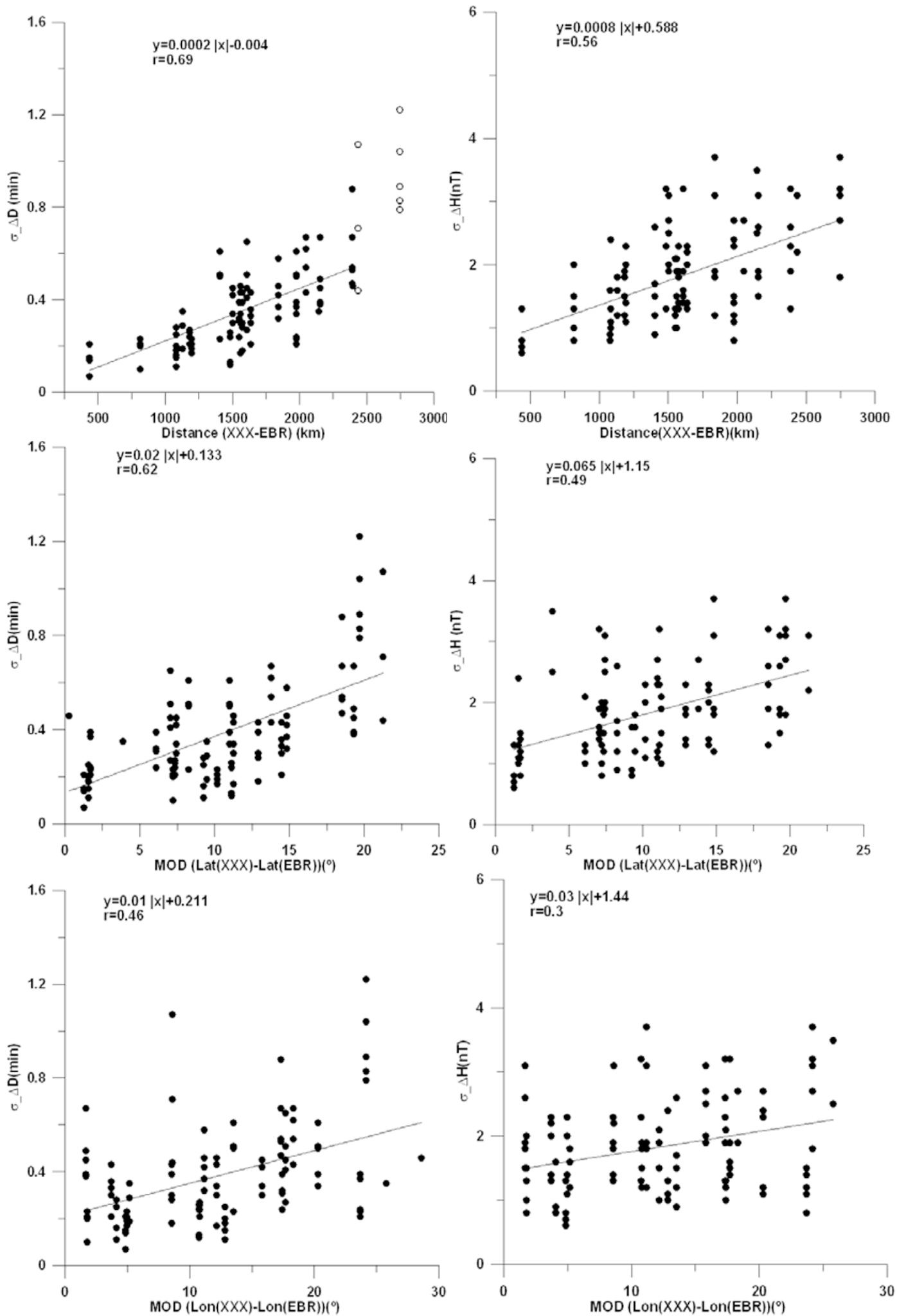

Fig. 7(a). $\sigma_{-} \Delta D$ (left) and $\sigma_{-} \Delta H$ (right) against distance and absolute value of latitude and longitude with respect to Ebro. Open circles are neglected points belonging to subauroral observatories with disturbed $D$ component.

an extrapolation is made in order to evaluate the value corresponding to the reference observatory (in our case, Ebro). Let us define Ebro* as a virtual observatory whose measurements are obtained by extrapolation of the values from other observatories. Ideally, the axis intercept of that linear fit should be zero, because standard deviation of Ebro*-Ebro should be nil. But in fact the virtual measurement Ebro* has close characteristics to Ebro but not the same response. Thus different response to latitudinal or longitudinal processes originated in the auroral zone or in the equatorial zone would affect this term.

In order to evaluate the degree of dependence of each 

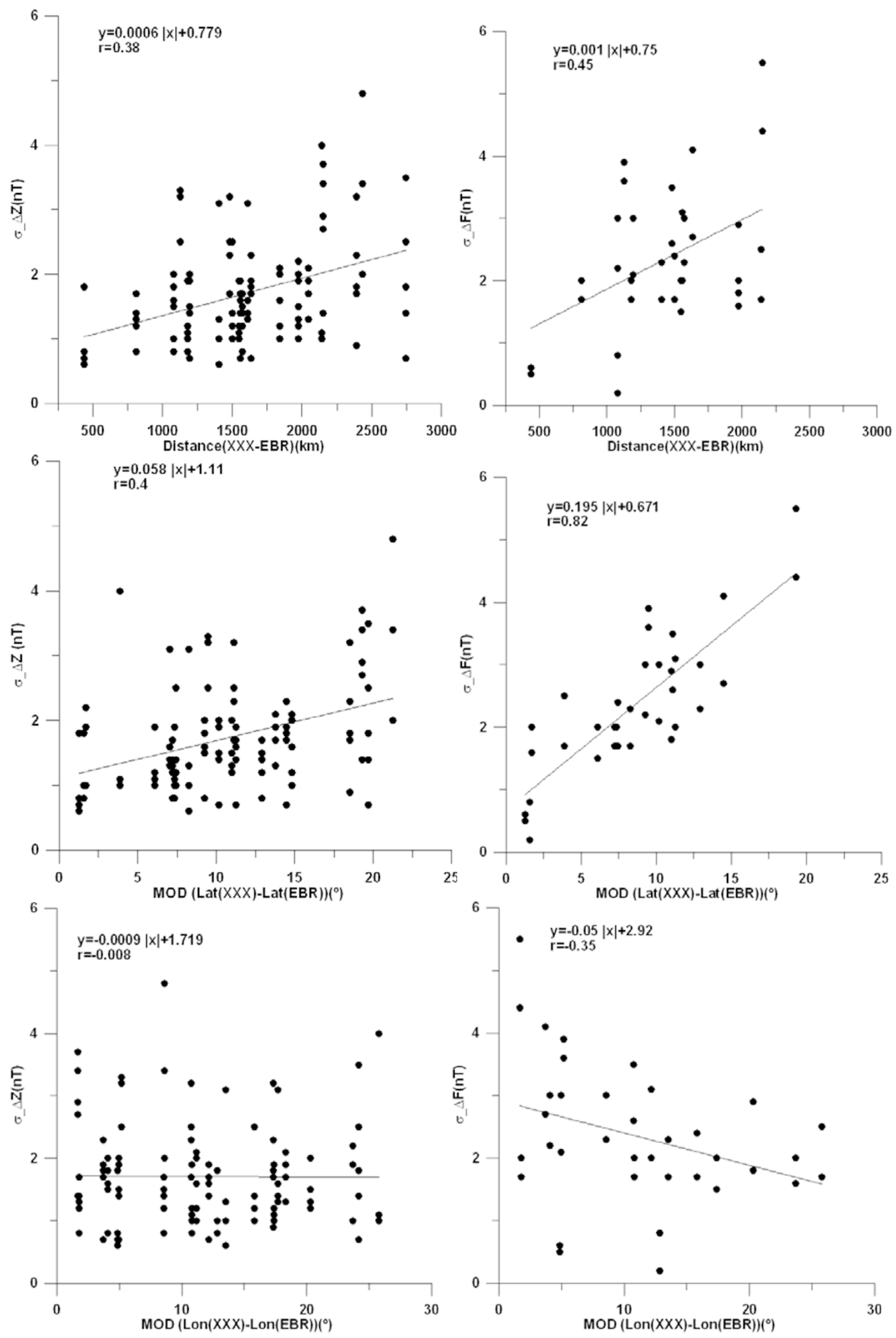

Fig. 7(b). $\sigma_{-} \Delta Z$ (left) and $\sigma_{-} \Delta F$ (right) against distance and absolute value of latitude and longitude with respect to Ebro.

magnetic element with the position variables, the linear correlation coefficient $(r)$ has been used. Visual inspection of Fig. 7 makes clear that linear regression has no meaning for the distance in some cases, and deserves discussion in the other cases. Small values of the intercept should be interpreted as good coherence of the observatory which has been taken as reference with the network (in this case EBR).

Some representations, such as $\sigma_{-} \Delta D$ (distance) or $\sigma_{-} \Delta F$ 
Table 2. Significance of the linear correlations according to their probabilities.

\begin{tabular}{ccccc}
\hline & $\sigma_{-} \Delta D$ & $\sigma_{-} \Delta H$ & $\sigma_{-} \Delta Z$ & $\sigma_{-} \Delta F$ \\
$(110$ points $)$ & $(110$ points $)$ & $(111$ points $)$ & $(36$ points $)$ \\
\hline DIST & $<0.05 \%\left(^{*}\right)$ & $<0.05 \%$ & $<0.05 \%$ & $1 \%$ \\
LAT & $<0.05 \%$ & $<0.05 \%$ & $<0.05 \%$ & $<0.05 \%$ \\
LONG & $<0.05 \%$ & $0.15 \%$ & $93 \%$ & $4 \%$ \\
\hline
\end{tabular}

$\left(^{*}\right)$ for this case, 102 points were used.
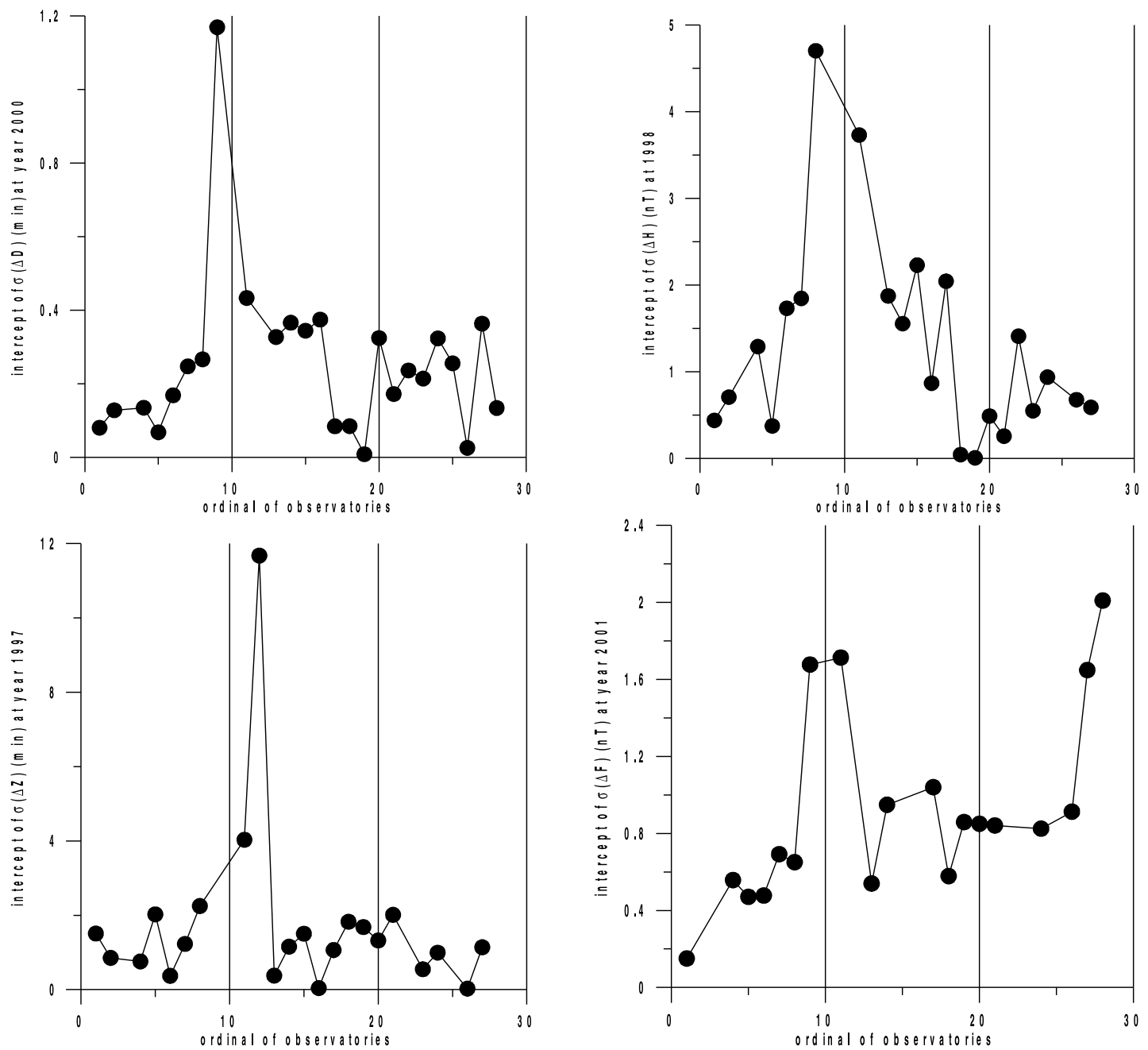

Fig. 8. Examples of annual mean values of the intercepts for the observatories participating in the test. Different components and years are shown. Several clear outlier points appear. Ordinal numbers relate to observatories in Table 1.

(latitude), describe a good linear fit, while others, as $\sigma_{-} \Delta H$ (longitude) or $\sigma_{-} \Delta Z$ (longitude), have a poorer fit. We conclude that $H$ and $Z$ are not linearly related to longitude, but are to latitude, hence many disturbances have a northsouth dependence rather than east-west.

Due to the peripheral location of EBR observatory with respect to the observatories within the network, the fits are highly dependent on the values of the extreme observatories, i.e., both those very close to EBR and those very far from EBR. This is the case for $D$, which would produce even a negative value of the intercept. In general, large intercepts should be interpreted as poor coherency of the observatory with the net, or malfunction of the sensors, as incorrect sensitivity or misalignment. Clearly northern observatories (NUR, DOM, LOV, ESK) are more affected by magnetospheric activity and especially by the ionospheric currents associated to the FAC's system. So, large networks are not convenient for this kind of study. Although the Wingst/Niemegk selection of the quiet days is done with care, in years close to the maximum of solar activity, as those on scope here, it is not possible to assure that those 10 days per month are absolutely quiet. For the case $\sigma_{-} \Delta D$ (distance) we decided not include northern observatories in the final set.

In order to choose the right value of the axis intercept and to be able to compare it to the rest of the observatories, it has been computed for each magnetic element. The value of the intercept with the ordinate axis differs according to which variable was considered. For example, $\sigma_{-} \Delta H$ has an ordinate in the origin of $0.6 \mathrm{nT}$ if it is expressed 

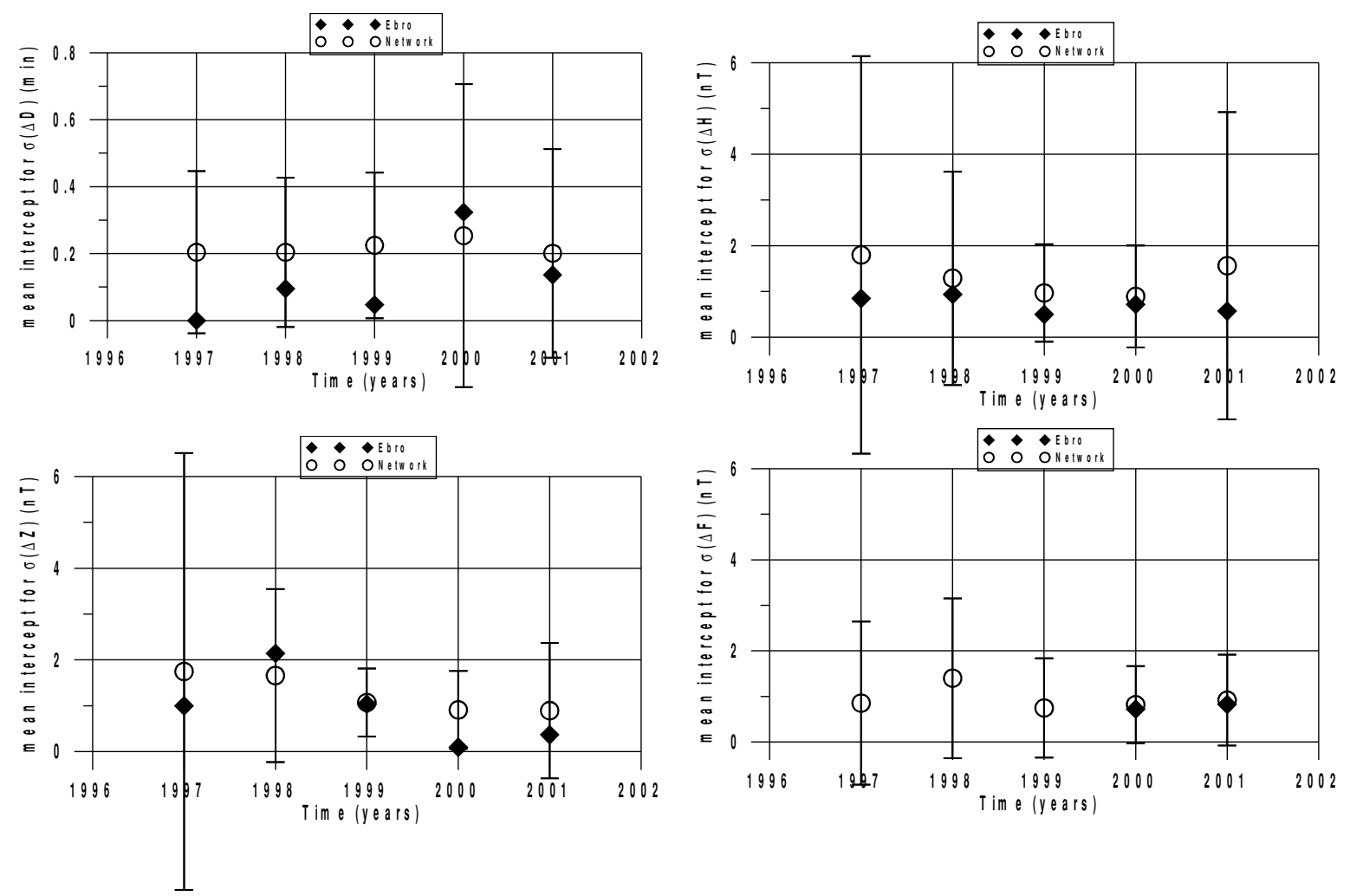

Fig. 9. Annual mean values of the intercepts of $D, H, Z$ and $F$ for Ebro against the corresponding values of the whole network for the quinquennium 1997-2001.

as a function of distance, $1.2 \mathrm{nT}$ when it is expressed as a function of latitude, and $1.4 \mathrm{nT}$ if it is expressed as a function of longitude.

To choose the most representative value for each element we have to consider not only its regression coefficient but also its probability of occurrence. With $N$ data points, this is defined as the probability to obtain a correlation coefficient equal to or greater than that being observed $\left(r_{o}\right)$ if these variables were not really correlated. Of course, the lower the probability, the higher the significance of the relationship between both variables is.

Table 2 is obtained by taking into account the number of data points and the correlation coefficient in each case (Taylor, 1982).

Probabilities less than $5 \%$ can be considered as significant, while those less $1 \%$ can be considered as very significant. Hence, most of the above relations are significant.

We can conclude that distance is a good variable in the case of declination and horizontal component, whereas latitude is a good variable in the case of vertical component and total field. The intercepts with the ordinate axis having the most significant correlation coefficients are:

$$
\begin{aligned}
& \sigma_{-} \Delta D(\mathrm{DIST}=0)=0.00 \pm 0.04 \mathrm{~min} \\
& \sigma_{-} \Delta H(\mathrm{DIST}=0)=0.6 \pm 0.2 \mathrm{nT} \\
& \sigma_{-} \Delta Z\left(\mathrm{LAT}=\mathrm{LAT}_{\mathrm{EBR}}\right)=1.1 \pm 0.1 \mathrm{nT} \\
& \sigma_{-} \Delta F\left(\mathrm{LAT}=\mathrm{LAT}_{\mathrm{EBR}}\right)=0.7 \pm 0.2 \mathrm{nT}
\end{aligned}
$$

The quality of the fits (expressed by their correlation coefficient) is equivalent to the previous one showing a good agreement between the independent techniques used.
2.2.2 Detection of outsiders With this method and taking the distance as the abscissa's variable, we can detect some anomalous values which considerably deviate from the whole net (Fig. 8), pointing out a necessary revision of these measurements.

2.2.3 Test of the performance of the whole network Evolution of the performance of the network in the preceding years (1973-1995) was shown by Schulz and Gentz (1998), detailing its increasing improvement. Intercomparison between observatory and international standards with the use of QHM magnetometers in the seventies and the generalization of the DI-flux magnetometers in the eighties (Bitterly, 1990) contributed to this advance.

Finally, comparison between yearly mean values of the intercepts for Ebro and the intercepts of the whole network are shown in Fig. 9.

Error bars represent twice the standard deviation which includes the $95 \%$ of the population as a representation of the majority of the network. Ebro results are always between these limits.

\section{Conclusions}

There is no evidence of discontinuities or jumps in Ebro magnetic elements for the period 1997-2001. The performance of the observatory is coherent with that of the nearby European observatories.

This kind of analysis gives better results if the observatory under study is central to the network and the size of the network is not too large, because the correlations are highly dependent on the coherence of the data, especially when there are phenomena with a small spatial scale. 
From a given observatory, direct comparison of its values with the mean value of the network is not adequate because there are gradients inside the inter-comparison zone which will give a different value depending on the situation of the set of observatories in the sample.

Sometimes, distance by itself is not the best variable to represent tendencies. Most of the disturbing phenomena: $\mathrm{SV}$, effects of auroral electrojet and ring currents have no radial symmetry but longitudinal or latitudinal distribution. In each case, longitudinal and latitudinal dependences have to be considered.

The selection of the ten quietest days per month does not guarantee that they are free from magnetic contamination from external sources.

Acknowledgments. Authors thank D. Gallart for his assistance in data managing and Dr. H. McCreadie for her language revision. Also they are grateful to Dr. G. Schulz and Dr. H. J. Linthe for their effort to provide these lists of quiet days and compile data from the observatories.

\section{References}

Bitterly, J., Declinometre-Inclinometre a Vanne de Flux, Institute de Physique du Globe de Strasbourg, 1990.

Bloxham, J. and D. Gubbins, The secular variation of Earth's magnetic field, Nature, 317, 777-781, 1985.
Curto, J. J. and E. Sanclement, Levelling error corrections to Dmeasurements in DI-flux magnetometers, Contributions to Geophysics \& Geodesy. Geophys. Inst. Slov. Acad. Sci., 31, 111-117, 2001.

Forbes, A. J., General Instrumentation, in Geomagnetism, edited by J. A. Jacobs, 51-136, Vol. 1, Academic Press, London, 1987.

Gaya-Piqué, L. R. and J. M. Torta, El Observatorio Geomagnético de Isla Livingston, Publicaciones del Observatorio del Ebro, Roquetes, 2000.

Jankowsky, J. and Ch. Sucksdorff, Guide for magnetic measurements and observatory practice, IAGA publications, Warsaw, 1996.

Macmillan, S. and S. Maus, International Geomagnetic Reference Field, the third generation, Earth Planets Space, 57, 1135-1140, 2005.

Marsal, S. and J. M. Torta, An evaluation of the uncertainty associated with the measurement of the geomagnetic field with a D/I fluxgate theodolite, Meas. Sci. Technol., 18, 2143-2156, 2007.

Parkinson, W. D., Introduction to Geomagnetism, Elsevier, 1983.

Schulz, G. and M. Beblo, Monitoring Geomagnetic Standards by the comparison of Momentary Values between European observatories in the past, present and future, VIth IAGA workshop on geomagnetic observatory instruments, data acquisition and processing, Dourbes, 1996.

Schulz, G. and I. Gentz, Results of the momentary value comparison between European observatories - a summary of the last two decades, VIIth IAGA workshop on geomagnetic observatory instruments, data acquisition and processing, Niemegk, 1998.

Stuart, W. F., Earth's Field Magnetometry, Rep. Prog. Phys., 838-847, 1972.

Taylor, J. R., An introduction to error analysis, the study of uncertainties in Physical Measurements, University Science Books, Oxford University Press, 1982.

J. J. Curto (e-mail: jjcurto@obsebre.es) and S. Marsal 